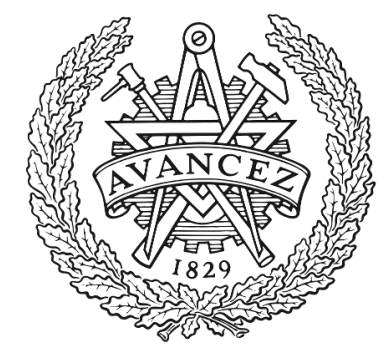

\title{
CHALMERS
}

UNIVERSITY OF TECHNOLOGY

\section{Broadband terahertz radiation from two-color mid- and far-infrared laser filaments in air}

Downloaded from: https://research.chalmers.se, 2023-04-26 11:43 UTC

Citation for the original published paper (version of record):

Nguyen, A., González De Alaiza Martínez, P., Thiele, I. et al (2018). Broadband terahertz radiation from two-color mid- and far-infrared laser filaments in air. Physical Review A, 97(6).

http://dx.doi.org/10.1103/PhysRevA.97.063839

N.B. When citing this work, cite the original published paper. 


\title{
Broadband terahertz radiation from two-color mid- and far-infrared laser filaments in air
}

\author{
Alisée Nguyen, ${ }^{1,}{ }^{*}$ Pedro González de Alaiza Martínez, ${ }^{2}$ Illia Thiele, ${ }^{3}$ Stefan Skupin, ${ }^{4}$ and Luc Bergé ${ }^{1}$ \\ ${ }^{1}$ CEA, DAM, DIF, 91297 Arpajon, France \\ ${ }^{2}$ Université Bordeaux - CNRS - CEA, Centre Lasers Intenses et Applications, UMR 5107, 33405 Talence, France \\ ${ }^{3}$ Department of Physics, Chalmers University of Technology, SE-412 96 Göteborg, Sweden \\ ${ }^{4}$ Institut Lumière Matière, UMR 5306 Université Lyon 1 - CNRS, Université de Lyon, 69622 Villeurbanne, France
}

(Received 27 February 2018; published 18 June 2018)

\begin{abstract}
We study terahertz (THz) emission by two-color femtosecond filaments in air using pump wavelengths $\lambda_{0}$ from 0.8 to $10.6 \mu \mathrm{m}$. Comprehensive three-dimensional numerical simulations show that tens-of-centimeter long filaments created by a $10.6-\mu \mathrm{m}$ pump pulse can produce $\mathrm{mJ}$ energy yields and $\mathrm{GV} / \mathrm{m}$ field strengths, while the laser-to-THz conversion efficiency exceeds the percent level. Changing temperature and humidity conditions reveals a surprisingly high stability of the $\mathrm{THz}$ spectra against different weather conditions. We also examine the role of many-body Coulomb effects on ionization. This additional plasma source promotes longer filaments that act as efficient $\mathrm{THz}$ emitters, despite enhanced plasma defocusing.
\end{abstract}

DOI: 10.1103/PhysRevA.97.063839

\section{INTRODUCTION}

Terahertz $(\mathrm{THz})$ science is attracting a broad interest because of its ability to detect many molecular fingerprints through noninvasive spectroscopy methods [1]. Efficient laserbased $\mathrm{THz}$ sources rely on the nonlinear polarization in $\chi^{(2)}$ crystals [2] or photocurrents in gas-plasma [3]. A well-known technique is to focus a two-color (2C) femtosecond light pulse, composed of a fundamental pulse (FH) and its second harmonic (SH), into air. From the resulting plasma, THz radiation develops high field strengths and broad spectra, even remotely [4] with no limitation due to laser damage. Recent studies [5,6] showed that increasing the pump wavelength can enhance the $\mathrm{THz}$ conversion efficiency (CE), which is at most limited to $\sim 10^{-4}$ for a $0.8-\mu \mathrm{m} 2 \mathrm{C}$ pump pulse. Whereas optical rectification in large-size organic crystals allows one to achieve CEs up to the percent level [2], the THz fluence remains below the $\mathrm{mJ} / \mathrm{cm}^{2}$ range. To exceed the $\mathrm{mJ}$ limit from small interaction surfaces, increasing the value of the laser wavelength may supply an efficient alternative. Longer laser wavelengths, e.g., $3.9 \mu \mathrm{m}$ delivered by optical parametric chirped pulse amplification, are nowadays accessible in the femtosecond regime [7]. Ultrafast (100 fs) $\mathrm{CO}_{2}$ lasers operating at $10.6 \mu \mathrm{m}$ with energies close to the Joule level are also expected in the coming years [8,9]. Therefore, long-range numerical simulations using energetic, sub-ps pulses have been performed in these wavelength domains $[10,11]$. They revealed, among others, the possibility to enhance the nonlinear propagation path by copropagating higher harmonics created through carrier wave shock formation [12].

In this paper, we focus on intense, broadband $\mathrm{THz}$ fields created by long-wavelength pulses. By means of a threedimensional (3D) unidirectional solver [13], we investigate $\mathrm{THz}$ generation in air by $2 \mathrm{C}$ femtosecond pulses. The pump wavelength $\lambda_{0}$ is varied from 0.8 to $10.6 \mu \mathrm{m}$. Our numerical

\footnotetext{
*alisee.nguyen@cea.fr
}

results display evidence of unprecedented $\mathrm{THz}$ energy yields and CEs achieved over long distances, in agreement with local current (LC) estimations [14]. For $\mathrm{CO}_{2}$ lasers, we also show that the weather conditions have a surprisingly little impact on the THz spectra. Besides the standard photo-induced field ionization, we finally account for many-body Coulomb ionization in argon, expected to become significant at $10-\mu \mathrm{m}$ pump wavelength $[15,16]$. This additional plasma source efficiently contributes to $\mathrm{THz}$ generation earlier in the pulse history.

\section{LOCAL CURRENT EVALUATIONS}

To start with, we use the LC model [14] to evaluate the performances of long-wavelength optical pulses as potential drivers for $\mathrm{THz}$ emission. Here, the THz field $E_{\mathrm{THz}}$ is extracted from filtering the secondary field $E_{J} \propto \partial_{t} J$ emitted from the free electron current density $J$ [17]. The filtering frequency is chosen as $v_{f}=10 \mathrm{THz}$, in order to limit the influence of the pump broadening into the $\mathrm{THz}$ spectrum. At moderate laser intensities $<10^{15} \mathrm{~W} / \mathrm{cm}^{2}$, the current density is given by a plasma fluid model [3], $\partial_{t} J+v_{c} J=e^{2} N_{e} E / m_{e}$, where $e$ and $m_{e}$ are the electron charge and mass, respectively; $v_{c} \approx 2.86 \mathrm{ps}^{-1}$ is the electron-neutral collision rate [18]. $N_{e}$ is the density of free electrons governed by

$$
\partial_{t} N_{e}=\sum_{i} W_{i}(E)\left(N_{a, i}-N_{e, i}\right),
$$

where $N_{a, i}$ and $N_{e, i}$ are the initial gas density of species $i=\mathrm{O}_{2}, \mathrm{~N}_{2}$, and their extracted electron density, respectively, so that $N_{e}=\sum_{i} N_{e, i} . W_{i}(E)$ is the field-dependent version of the cycle-averaged Perelomov, Popov and Terent'ev (PPT) ionization rate [19] applied for air to $80 \%$ of nitrogen and $20 \%$ of oxygen with ionization potentials $U_{i}\left(\mathrm{~N}_{2}\right)=15.6 \mathrm{eV}$ and $U_{i}\left(\mathrm{O}_{2}\right)=12.1 \mathrm{eV}$. For argon we will use $U_{i}(\mathrm{Ar})=15.76 \mathrm{eV}$.

We apply the LC model to the local input laser field

$$
E(t)=E_{0} \sum_{n=1,2} a_{n} e^{-\left(2 n \ln 2 \frac{t}{\tau}\right)^{2}} \cos \left(n \omega_{0} t+\phi_{n}\right),
$$



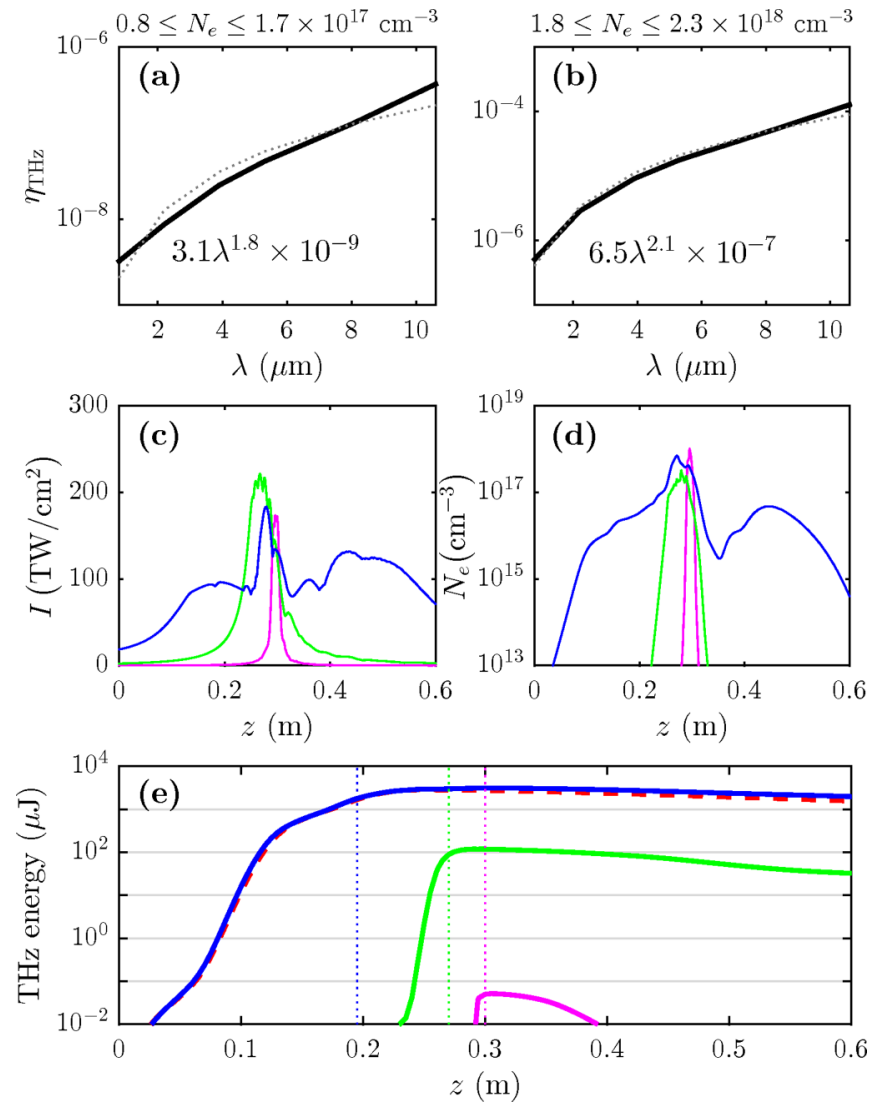

FIG. 1. (a), (b) Laser-to-THz CE evaluated in the frequency window $v<10 \mathrm{THz}$ from the LC model using the two input fluences (a) $5 \mathrm{~J} / \mathrm{cm}^{2}$ and (b) $10 \mathrm{~J} / \mathrm{cm}^{2}\left(\phi_{1}=0, \phi_{2}=\pi / 2\right)$ and producing electron densities that barely vary with the $\mathrm{FH}$ wavelength (see values indicated on the top). Dotted gray curves are fits in $\lambda^{\alpha}$. (c), (d), and (e) Results from 3D UPPE simulations of 2C near- to far-infrared filaments. (c) Maximum intensity along $z$ using winter HITRAN dispersion for FH pump wavelengths of 0.8 (magenta/dark gray), 3.9 (green/light gray), and $10.6 \mu \mathrm{m}$ (blue/black curves). Associated (d) peak electron densities and (e) THz energies [integral over $(x, y, t)]$, computed along $z$ for winter (upper solid blue) and summer (dashed red curve) in the case of the 10.6- $\mu \mathrm{m} \mathrm{FH}$. Vertical dotted lines show the distances of maximum $\mathrm{THz}$ generation (see text).

where the amplitude $E_{0}=\sqrt{2 I_{0} / c \epsilon_{0}}$ depends on the pump intensity $I_{0}, \tau=v_{f}^{-1}=100 \mathrm{fs}$ is the full-width-at-halfmaximum (FWHM) pulse duration, $\omega_{0}=2 \pi c / \lambda_{0}$ is the $\mathrm{FH}$ frequency $(n=1), a_{n}$ and $\phi_{n}$ are the amplitude coefficient and phase of the $n$th harmonic, respectively. We consider $2 \mathrm{C}$ pulses with amplitude coefficients $a_{1}=\sqrt{0.9}, a_{2}=\sqrt{0.1}$ and laser fluences $U_{L}=5-10 \mathrm{~J} / \mathrm{cm}^{2}$, corresponding to $I_{0}=$ 100-200 TW $/ \mathrm{cm}^{2}$ peak intensities. The relative phase between the two colors is set to its optimum value of $\pi / 2$ for $\mathrm{THz}$ generation by photocurrents $[3,6]$. We show in Figs. 1(a) and 1(b) the evolution of the laser-to-THz CE $\eta_{\mathrm{THz}}=$ $\int_{-v_{f}}^{+v_{f}}\left|\widehat{\partial_{t} J}\right|^{2} d v /\left[\left(e^{2} N_{a} / m_{e}\right)^{2} U_{L}\right]$ with the pump wavelength [20], for which we compute the electron density through Eq. (1) for $\mathrm{O}_{2}$. With a $10.6-\mu \mathrm{m}$ pump wavelength, a gain of about two orders of magnitude can be expected in the THz energy yield compared with a near-IR pump. A tentative scaling in $\lambda^{\alpha}$ supports an exponent $\alpha$ close to 2 [6]. The achievable
TABLE I. Pump wavelengths, Kerr indices, Raman-delayed ratios, and input laser energies for air and argon.

\begin{tabular}{lcccc}
\hline \hline$\lambda_{0}(\mu \mathrm{m}) /$ gas & $0.8 /$ air & $3.9 /$ air & $10.6 /$ air & $10 /$ argon \\
\hline$n_{2}\left(10^{-19} \mathrm{~cm}^{2} / \mathrm{W}\right)$ & 3.8 & 3.6 & 3.3 & 1.0 \\
$x_{K}$ & 0.8 & 0.8 & 0.8 & 0.0 \\
$U_{L}(\mathrm{~mJ})$ & 0.494 & 12.4 & 100 & 169.4 \\
\hline \hline
\end{tabular}

$\mathrm{THz}$ energy should thus be readily increased by four orders of magnitude when multiplying the $\mathrm{FH}$ wavelength by a factor 10, first through photocurrents (Fig. 1), second by preserving in the pump beam the same amount of critical powers for self-focusing $P_{\mathrm{cr}} \propto \lambda_{0}^{2}$. This latter scaling law invites us to operate in the filamentation regime [21], for which the larger critical power at long wavelengths renders the self-guiding of high pump energies in a single filament possible, without beam breakup.

\section{UPPE SIMULATIONS}

To test the previous estimates, we use the 3D unidirectional pulse propagation equation (UPPE) [13]:

$$
\partial_{z} \widehat{E}=i \sqrt{k^{2}(\omega)-k_{x}^{2}-k_{y}^{2}} \widehat{E}+i \frac{\mu_{0} \omega^{2}}{2 k(\omega)} \widehat{\mathcal{F}}_{\mathrm{NL}},
$$

where $k(\omega)=n(\omega) \omega / c$ is the wave number with air refraction index $n(\omega), z$ is the propagation variable, and $\widehat{E}\left(k_{x}, k_{y}, z, \omega\right)$ is the Fourier transform of the electric field. The first term on the right-hand side of Eq. (3) describes linear dispersion and diffraction of the pulse. The second term $\widehat{\mathcal{F}}_{\mathrm{NL}}$ contains the third-order nonlinear polarization $\widehat{P}_{\mathrm{NL}}$, the electron current $\widehat{J}$, and a loss term due to ionization [21]. Air dispersion is taken from [22], completed with HITRAN data [23,24] accounting for water vapor and carbon dioxide. The main absorption lines are located at 2.7 and 5.2-7.3 $\mu \mathrm{m}$ for $\mathrm{H}_{2} \mathrm{O}$ and $4.2 \mu \mathrm{m}$ for $\mathrm{CO}_{2}$. Two ground-level weather configurations are examined: either "winter" using a moderate temperature of $15^{\circ} \mathrm{C}$ and local humidity of $20 \%$, or "summer" with $25^{\circ} \mathrm{C}$ temperature and $60 \%$ humidity. The Kerr index $n_{2}$ entering $P_{\mathrm{NL}}$ is given in Table I with Raman-delayed response ratio $x_{K}[25,26]$. The number of critical powers, $P_{\mathrm{cr}}=\lambda_{0}^{2} / 2 \pi n_{2}$, is kept constant and set to 1.73. Plasma generation proceeds from photoionization initiated by the PPT rate [Eq. (1)], supplemented by the avalanche ionization term $\mu_{0} c e^{2} v_{c} I N_{e} /\left[U_{i} m_{e}\left(v_{c}^{2}+\omega^{2}\right)\right]$, which originates from collisions between free electrons and neutrals currently associated with inverse Bremsstrahlung.

In our simulations, the input light field $(z=0)$ reads as Eq. (2) multiplied by a transverse Gaussian profile with $1 / e^{2}$ radius of $2.5 \mathrm{~mm}$ that is focused by a converging lens of $30-\mathrm{cm}$ focal length. The initial phases $\phi_{1}$ and $\phi_{2}$ are set to zero as the phase angle is continuously evolving through Gouy-phase shift and nonlinear propagation effects. Input pulse energies are indicated in Table I. Numerical simulations were performed using a $15 \times 15 \mathrm{~mm}^{2}$ transverse box and a $\sim 4.3 \mathrm{ps}$ temporal window. As seen from Figs. 1(c) and 1(d), peak intensities of $\sim 200 \mathrm{TW} / \mathrm{cm}^{2}$ produce electron densities above $10^{17} \mathrm{~cm}^{-3}$. When increasing the pump wavelength, longer filaments form (see also [27]). Due to the smaller Rayleigh range, the 10.6- $\mu \mathrm{m}$ 

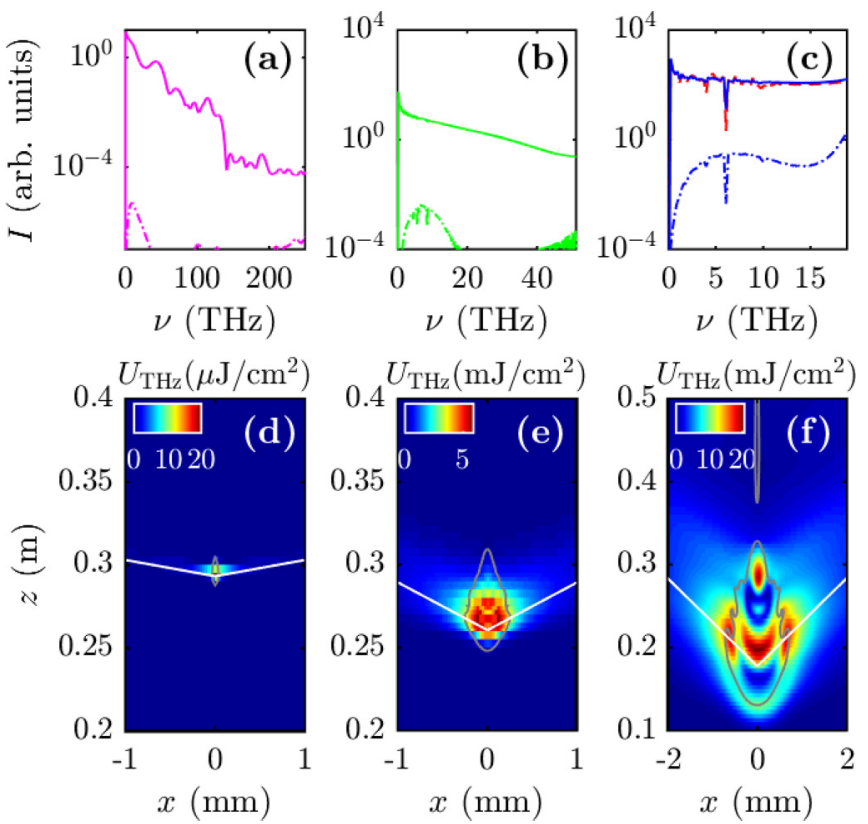

FIG. 2. (a), (b), and (c) Transversally averaged THz spectra at distances of maximum THz generation (a) $z=30 \mathrm{~cm}$, (b) $z=27 \mathrm{~cm}$, and (c) $z=19.5 \mathrm{~cm}$ using the same color plot style as in Figs. 1(c)1(e). Dash-dotted curves show the same quantities without plasma generation. (d)-(f) $\mathrm{THz}$ pulse fluence in the $(x, z)$ plane for $\mathrm{FH}$ pump wavelengths of (d) 0.8 , (e) 3.9 , and (f) $10.6 \mu \mathrm{m}$. White lines serve as eye guides for the conical emission angles, fitting the curve $z_{\max }(x)$ such that $U_{\mathrm{THz}}\left(x, z_{\max }\right)=\max _{z} U_{\mathrm{THz}}(x, z)$. Plasma transverse isocontours at $10^{16} \mathrm{~cm}^{-3}$ are indicated by gray lines.

pump pulse starts its self-focusing sequence earlier. Figure 1(e) illustrates the THz energy inside the numerical box versus the propagation axis. This energy remains below the $\mu \mathrm{J}$ level for $\lambda_{0}=0.8 \mu \mathrm{m}$, but it increases to the $0.1 \mathrm{~mJ}$ level for mid-IR pumps and even to $3.1 \mathrm{~mJ}$ for far-IR lasers, confirming the 4 orders of magnitude increase expected from the LC model above. The $\mathrm{THz}$ CE obtained at $10.6 \mu \mathrm{m}$ is $3 \%$ against $1 \%$ at
$3.9 \mu \mathrm{m}$. Let us notice that the yield curves of Fig. 1(e) decrease instead of saturating to a plateau value, which is mainly due to $\mathrm{THz}$ components leaving our finite time window.

To go one step further, Figs. 2(a), 2(b), and 2(c) detail the $\mathrm{THz}$ spectra computed at the distance of maximum $\mathrm{THz}$ production, for which the slope of the THz energy in Fig. 1(e) is the steepest. Because diffraction of $\mathrm{THz}$ pulses is very fast, this distance corresponds in most of cases to the location of the maximum on-axis $\mathrm{THz}$ intensity. Apart from the impressive increase in the $\mathrm{THz}$ spectrum, Fig. 2(c) also evidences for the $10.6 \mu \mathrm{m}$ pump pulse that augmenting humidity and temperature makes water absorption lines stronger around 3 and $6 \mathrm{THz}$. However, these absorption zones are spectrally narrow and they do not visibly reduce the total $\mathrm{THz}$ yield, as confirmed by Fig. 1(e). Besides, an important debate has been the role of Kerr-induced four-wave mixing compared to that of photocurrents in laser-driven $\mathrm{THz}$ pulse generation [18]. To clear up this point, Figs. 2(a), 2(b), and 2(c) compare $\mathrm{THz}$ spectra computed until the indicated distances without the plasma terms. The presence of plasma clearly enlarges the spectra by about 3 orders of magnitude and shifts the peak frequencies down to $v_{\mathrm{THz}}<1 \mathrm{THz}$. Hence, plasma generation and related photocurrents - even involving rather low electron densities $\sim 10^{15-17} \mathrm{~cm}^{-3}$ - remain the key players in $\mathrm{THz}$ pulse generation. Figures 2(d), 2(e), and 2(h) evidence the broader laser-plasma interaction surfaces engaged at longer FH wavelengths. This is due to the $\lambda_{0}$-dependent increase in the filament waist [27,28], and thus in the plasma transverse cross section, which amplifies in turn the THz yield. Also, the conical emission angle of the radiated $\mathrm{THz}$ pulses decreases with the FH wavelength: THz waves indeed diffract with angles of $6.2^{\circ}$ and $2.3^{\circ}$ for $\lambda_{0}=0.8$ and $3.9 \mu \mathrm{m}$, respectively. With a 10.6- $\mu \mathrm{m}$ pump pulse, in contrast, $\mathrm{THz}$ waves are emitted nearly on axis within a $1.2^{\circ}$ angle and they offer a better directivity.

Figure 3 displays the entire optical spectrum developed for the three FH wavelengths along the propagation axis, together with optical and $\mathrm{THz}$ field patterns at the distance of maximum
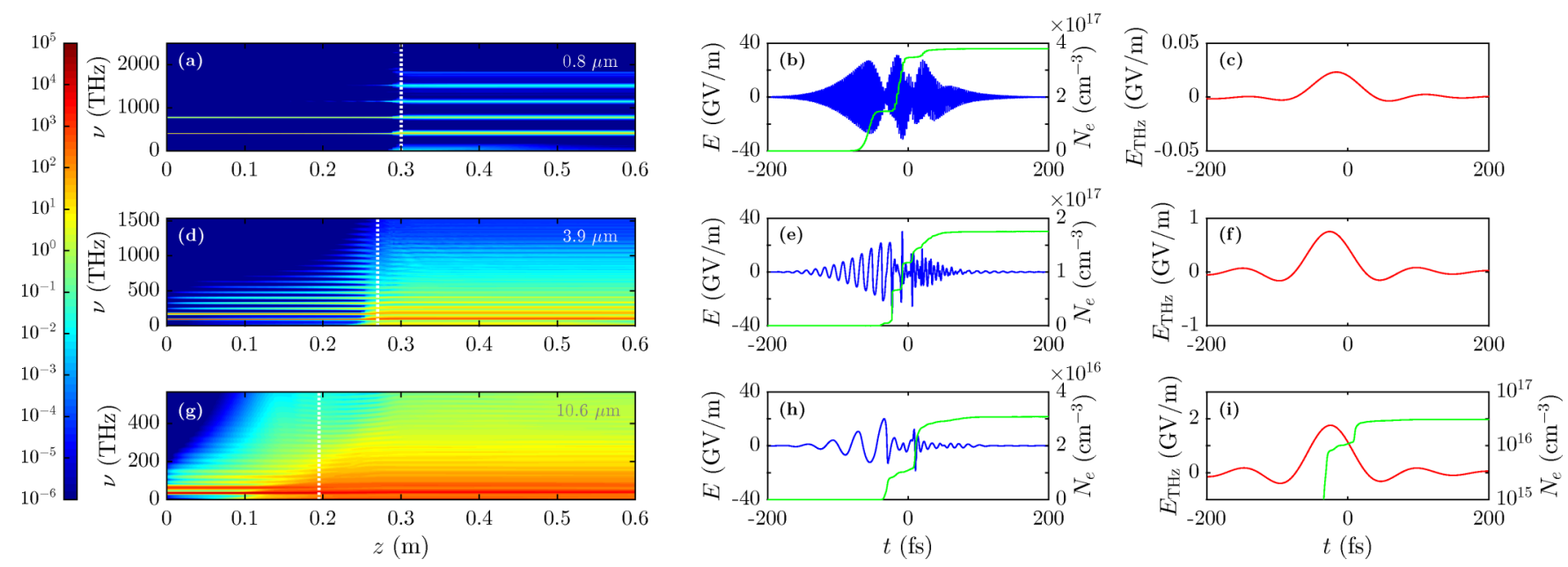

FIG. 3. (a), (d), and (g) Transversally averaged intensity spectra (left colored patterns) along propagation for $\lambda_{0}=$ (a) $0.8 \mu \mathrm{m}$, (d) $3.9 \mu \mathrm{m}$, and (g) $10.6 \mu \mathrm{m}$. The vertical dotted lines indicate the distance of maximum THz production. (b), (e), and (h) Corresponding on-axis propagating field (blue/black curve, left axis) and electron density (green/light gray curve, right axis) at maximum THz production. (c), (f), and (i) On-axis THz field obtained by filtering the total electric field $E$ with a 20th-order Butterworth filter in the frequency window $v<10$ THz. 


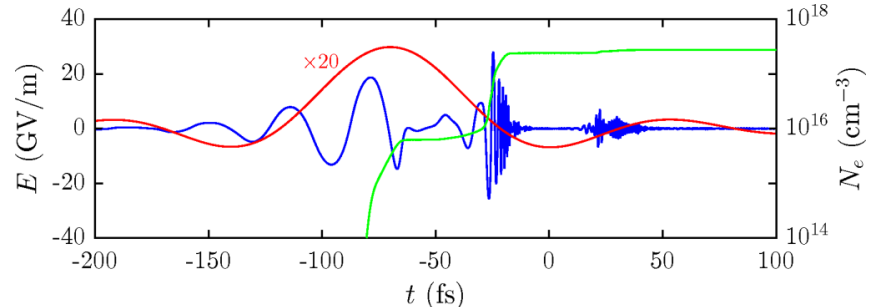

FIG. 4. On-axis electric field for the 10.6- $\mu \mathrm{m}$ pump pulse at $z=30 \mathrm{~cm}$ (blue/black curve, left axis). The red (dark gray) curve corresponds to the on-axis THz field (strength: $\times 20$ ). The green (light gray) curve shows related electron density (right axis).

$\mathrm{THz}$ generation. For a near-IR pump, this distance coincides with that of maximum plasma generation, close to focus. When increasing the pump wavelength, high-order harmonics are amplified over a longer coherence length [29] and they form a broader supercontinuum through the plasma nonlinearity, self-phase modulation and cross-phase modulation [Figs. 3(a), $3(\mathrm{~d})$, and $3(\mathrm{~g})]$. Ionization arises in the rear pulse, but intense pump components remain in the undepleted front pulse [see Figs. 3(b), 3(e), and 3(h)]. The THz field is peaked where the pump field is maximum and it increases with $\lambda_{0}$ [Figs. 3(c), 3(f), and 3(i)].

Another phenomenon appears at $10.6 \mu \mathrm{m}$. As suggested by Figs. 1(d) and 1(e), THz fields are maximum well before maximum plasma generation sets in. At focus, high harmonics mainly go on feeding ionization. However, they do not produce $\mathrm{THz}$ waves by photocurrents because their temporal profiles cannot form an efficient, asymmetric field triggering lowfrequency radiation. Figure 4 confirms this point: at focus the rear part of the electric field composed of the highest harmonics is intense enough to contribute alone to plasma generation. Ionization steps are created on axis by high harmonics that can propagate into the plasma, unlike the $\omega_{0}$ and $2 \omega_{0}$ optical components. However, THz generation still proceeds from the residual two colors surviving in the front pulse and forming early density steps. Because the broadest plasma surface extends at $z=19.5 \mathrm{~cm}$ [Fig. 2(f)], maximum THz generation takes place at this distance. Much less THz energy is produced at $z=30 \mathrm{~cm}$, as the plasma diameter is decreased by a factor $\sim 3.5$.

\section{MANY-BODY COULOMB IONIZATION}

Finally, it is compulsory to examine the many-body Coulomb ionization (MBI) effect on $\mathrm{THz}$ generation proposed by Schuh et al. $[15,16]$. Under strong laser field irradiation, excited-state electrons from neighboring atoms are able to collide and thus increase the ionization rate. This effect is all the more relevant as the pump wavelength is large. It manifests at low laser intensities where multiphoton or tunnel ionization vanishes. Therefore, it should be taken into account for far-infrared optical fields. To model it, we use the fit proposed in [16] for a $10-\mu \mathrm{m}$ pump wavelength interacting with argon atoms:

$$
W_{\mathrm{MBI}}(E)=C E^{4} \sqrt{\frac{E^{2}+s}{E^{2}}},
$$
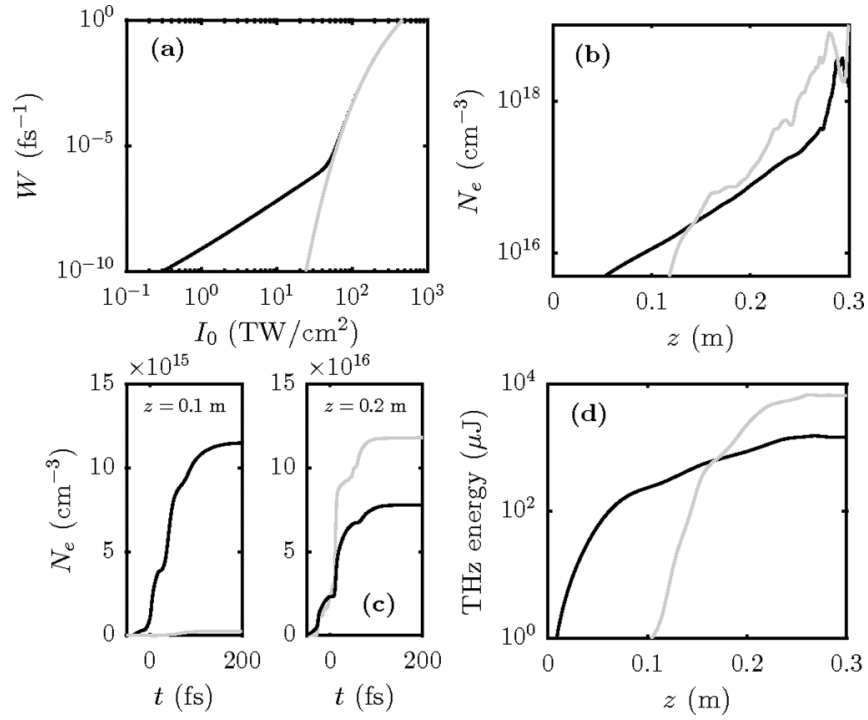

FIG. 5. Ionization rate vs laser intensity with (black curves) or without (gray curves) MBI evaluated from Eq. (2) as input in a 1-bar argon gas. (b) Peak electron density reached along propagation (UPPE simulations with collisional ionization) including (black) or not (gray curves) MBI. (c) Plasma density profiles in time at $z=0.1 \mathrm{~m}$ and $z=0.2 \mathrm{~m}$. (d) Associated THz energy yields.

where $C=1.1095 \times 10^{4} \mathrm{~m}^{4} / \mathrm{GV}^{4}$ and $s=4.6 \mathrm{GV}^{2} / \mathrm{m}^{2}$. So, we now turn to an argon gas at ambient pressure and, using Table I's parameters, simulate 100 -fs focused pump pulses operating at critical power with a $1 / e^{2}$ radius of $3.5 \mathrm{~mm}$. The MBI rate is added to the PPT rate of Eq. (1), yielding the ionization curve plotted in Fig. 5(a). Compared with the PPT rate alone, the MBI rate $\propto E^{4}$ is important in the intensity region $<20 \mathrm{TW} / \mathrm{cm}^{2}$ and allows a more efficient ionization at earlier propagation [see Fig. 5(b)]. Figure 5(c) details the plasma response in time, which is mainly governed by Eq. (4) in the range $z \leqslant 10 \mathrm{~cm}$. This exhibits a steplike increase, which can promote efficient photocurrents although the MBI steps are less sharp than the ones occurring in the tunnel regime [14] at, e.g., $z=20 \mathrm{~cm}$. With such plasma contribution, we finally report in Fig. 5(d) that THz emission starts at the very beginning of the propagation range, but it saturates due to accumulated plasma defocusing actions. Without MBI, a THz energy yield of $7 \mathrm{~mJ}$ is reached, corresponding to $4 \%$ conversion efficiency. With MBI, comparable, although quantitatively smaller values are reached $(1.5 \mathrm{~mJ} \mathrm{THz}$ energy and $0.9 \% \mathrm{CE})$.

\section{CONCLUSION}

In summary, we demonstrated by means of numerical simulations that two-color $\mathrm{CO}_{2}$ laser filaments can provide $\mathrm{THz}$ conversion efficiencies above the percent level at moderate laser intensities. Far-infrared filaments are able to self-channel over longer distances and supply $\mathrm{THz}$ energy yields of several $\mathrm{mJ}$. We outlined the effects of weather conditions as well as the influence of many-body ionization. Even if the latter effects may mitigate the conversion efficiency to some extent, they still enable to produce $\mathrm{mJ}$-energetic $\mathrm{THz}$ fields over long propagation ranges. 


\section{ACKNOWLEDGMENTS}

This work was supported by the ANR/ASTRID Project "ALTESSE" No. ANR-15-ASTR-0009 and performed using
HPC resources from GENCI (Grant No. A0020507594). S.S. acknowledges support by the Qatar National Research Fund (Grant No. NPRP 8-246-1-060).
[1] M. Tonouchi, Nat. Photon. 1, 97 (2007).

[2] C. Vicario, B. Monoszlai, and C. P. Hauri, Phys. Rev. Lett. 112, 213901 (2014).

[3] K. Y. Kim, A. J. Taylor, J. H. Glownia, and G. Rodriguez, Nat. Photon. 2, 605 (2008).

[4] J.-F. Daigle, F. Théberge, M. Henriksson, T.-J. Wang, S. Yuan, M. Châteauneuf, J. Dubois, M. Piché, and S. L. Chin, Opt. Express 20, 6825 (2012).

[5] M. Clerici, M. Peccianti, B. E. Schmidt, L. Caspani, M. Shalaby, M. Giguère, A. Lotti, A. Couairon, F. Légaré, T. Ozaki, D. Faccio, and R. Morandotti, Phys. Rev. Lett. 110, 253901 (2013).

[6] A. Nguyen, P. González de Alaiza Martínez, J. Déchard, I. Thiele, I. Babushkin, S. Skupin, and L. Bergé, Opt. Express 25, 4720 (2017).

[7] D. Kartashov, S. Ališauskas, A. Pugžlys, A. Voronin, A. Zheltikov, M. Petrarca, P. Béjot, J. Kasparian, J.-P. Wolf, and A. Baltuška, Opt. Lett. 37, 3456 (2012).

[8] I. V. Pogorelsky, M. Babzien, I. Ben-Zvi, J. Skaritka, and M. N. Polyansky, Nucl. Instrum. Methods Phys. Res. A 829, 432 (2016).

[9] S. Y. Tochitsky, J. J. Pigeon, D. J. Haberberger, C. Gong, and C. Joshi, Opt. Express 20, 13762 (2012).

[10] N. A. Panov, D. E. Shipilo, V. A. Andreeva, O. G. Kosareva, A. M. Saletsky, H. Xu, and P. Polynkin, Phys. Rev. A 94, 041801 (2016).

[11] Y. E. Geints and A. A. Zemlyanov, Appl. Opt. 53, 5641 (2014).

[12] P. Panagiotopoulos, P. Whalen, M. Kolesik, and J. Moloney, Nat. Photon. 9, 543 (2015).

[13] M. Kolesik and J. V. Moloney, Phys. Rev. E 70, 036604 (2004).
[14] I. Babushkin, W. Kuehn, C. Köhler, S. Skupin, L. Bergé, K. Reimann, M. Woerner, J. Herrmann, and T. Elsaesser, Phys. Rev. Lett. 105, 053903 (2010).

[15] K. Schuh, J. Hader, J. V. Moloney, and S. W. Koch, Phys. Rev. E 89, 033103 (2014).

[16] K. Schuh, M. Kolesik, E. M. Wright, J. V. Moloney, and S. W. Koch, Phys. Rev. Lett. 118, 063901 (2017).

[17] O. D. Jefimenko, Electricity and Magnetism: An Introduction to the Theory of Electric and Magnetic Fields (Appleton-CenturyCrofts, New York, 1966).

[18] V. A. Andreeva, O. G. Kosareva, N. A. Panov, D. E. Shipilo, P. M. Solyankin, M. N. Esaulkov, P. González de Alaiza Martínez, A. P. Shkurinov, V. A. Makarov, L. Bergé, and S. L. Chin, Phys. Rev. Lett. 116, 063902 (2016).

[19] A. M. Perelomov, V. S. Popov, and M. V. Terent'ev, JETP (USSR) 50, 1393 (1966) [Sov. Phys. JETP 23, 924 (1966)].

[20] A. Nguyen, P. González de Alaiza Martínez, I. Thiele, S. Skupin, and L. Bergé, New J. Phys. 20, 033026 (2018).

[21] L. Bergé, S. Skupin, R. Nuter, J. Kasparian, and J. P. Wolf, Rep. Prog. Phys. 70, 1633 (2007).

[22] E. R. Peck and K. Reeder, J. Opt. Soc. Am. A 62, 958 (1972).

[23] Hitran on the Web, http://hitran.iao.ru/

[24] L. S. Rothman et al., J. Quant. Spectrosc. Radiat. Transfer 130, 4 (2013), HITRAN2012 special issue.

[25] S. Zahedpour, J. K. Wahlstrand, and H. M. Milchberg, Opt. Lett. 40, 5794 (2015).

[26] J. J. Pigeon, S. Y. Tochitsky, E. C. Welch, and C. Joshi, Opt. Lett. 41, 3924 (2016).

[27] Y. E. Geints and A. A. Zemlyanov, Appl. Opt. 56, 1397 (2017).

[28] L. Bergé, Opt. Express 16, 21529 (2008).

[29] L. Bergé, J. Rolle, and C. Köhler, Phys. Rev. A 88, 023816 (2013). 